\title{
Reproducibilidad del posicionamiento de pacientes en tratamiento con radioterapia del Centro Javeriano de Oncología
}

\author{
Ricardo A. Sánchez, MD ${ }^{1}$, Andrea GonzÁlez, Tnlgi ${ }^{2}$, Andrea Rocha, Tnlg ${ }^{2}$
}

\section{Resumen}

Introducción. Se encuentran incertidumbres por posicionamiento en los pacientes durante el tratamiento de radioterapia. Este estudio se realizó con la finalidad de mostrar la experiencia en nuestro centro.

Métodos. Se analizaron 316 pacientes en el periodo comprendido entre el 17 de marzo y el 18 de junio de 2010, y se compararon las radiografías digitales reconstruidas, Digitally Reconstructed Radiograph, DRR) con las radiológicas, en película radiográfica o imagen digital, y se hicieron las correcciones correspondientes en los tres ejes.

Resultados. Se registraron 809 imágenes en los 316 pacientes, la mayoría de cáncer de próstata. En $91 \%$ de los casos, las imágenes obtenidas no requirieron correcciones por posicionamiento durante el tratamiento. El promedio de correcciones en los tres ejes (X, Y y Z) fue de 3,4 $\mathrm{mm}$. Sin embargo, se presentaron correcciones de $5 \mathrm{~mm}$ o más en pacientes con obesidad.

Conclusión. En este estudio se encontró una adecuada reproducibilidad en nuestros pacientes durante el tratamiento, con incertidumbres de posicionamiento similares a las reportadas en la literatura. La mayoría se presentó en pacientes tratados por cáncer de próstata. Debe prestarse especial atención en los pacientes obesos o muy delgados, los cuales posiblemente requieren una mejor técnica de inmovilización.

Palabras clave: posicionamiento, radioterapia conformal, radiografía digital, inmovilización, cáncer de próstata.

1 Centro Javeriano de Oncología, Hospital Universitario San Ignacio. Profesor de la Facultad de Medicina, Pontificia Universidad Javeriana, Bogotá, D.C., Colombia.

2 Centro Javeriano de Oncología, Hospital Universitario San Ignacio. 
Title

Reproducibility of patient positioning in radiation therapy Centro Javeriano de Oncología

\begin{abstract}
Introduction: Positioning uncertainties are found in patients during treatment of radiotherapy. This study was conducted in order to show the experience in our center.
\end{abstract}

Methods: 316 patients were analyzed in the period from March 17 to June 18, 2010, which compared the images DRR (Digitally Reconstructed Radiographs) with radiological images either by X-ray film or digital image for performing corrections in the three axes.

Results: 809 images were recorded in 316 patients, mostly in prostate cancer. En $91 \%$ of the images made no corrections required during the treatment, positioning corrections in all three axes $(\mathrm{X}, \mathrm{Y}, \mathrm{Z})$ was $3.4 \mathrm{~mm}$, however corrections of $5 \mathrm{~mm}$ or more in obesity patients was found.

Conclusion: In this study we found a suitable reproduction for our patients during treatment with uncertainties positioning similar to those reported in the literature, the greater quantity was founded in patients treated with prostate cancer. Special attention should be paid in obese and very thin patients who may require improved immobilization technique.

Key words: positioning, conformal radiotherapy, digital radiography, immobilization, prostate cancer

\section{Introducción}

Durante el tratamiento de radioterapia existen incertidumbres sistemáticas y aleatorias durante la fase de planeación de administración del tratamiento. Las sistemáticas se deben a errores en la planeación y simulación, por ejemplo, diferente posicionamien- to del paciente durante la adquisición de las imágenes para la planeación y durante el posicionamiento del tratamiento. Los errores aleatorios aparecen por las desviaciones diarias al colocar el paciente en la mesa de tratamiento, o cambios anatómicos del tumor o por movimientos internos de los órganos.

Estas incertidumbres son las responsables de que exista el volumen blanco de planificación (planning target volume, PTV) que simplemente es un margen de seguridad adecuado para asegurar que el volumen blanco reciba la dosis prescrita (ICRU 50-62, International Commission on Radiation Units and Measurements 50-62).

El objetivo del presente estudio fue mostrar nuestra experiencia en los resultados de las incertidumbres por posicionamiento de los pacientes tratados con radioterapia.

\section{Materiales y métodos}

Este estudio se llevó a cabo en el periodo comprendido entre el 17 de marzo y el 18 de junio de 2010, para lo cual se tomó una muestra de 316 pacientes con diferentes enfermedades oncológicas del Departamento de Radioterapia del Centro Javeriano de Oncología, Hospital Universitario San Ignacio. Los datos tabulados se muestran en la tabla 1. 
Tabla 1. Total de pacientes por técnica $y$ proyecciones imagenológicas realizadas

\begin{tabular}{lrr}
\hline & & Total \\
\hline Mujeres & 161 & \\
Hombres & 155 & \\
Técnica 3D CRT & 241 & \\
Técnica IMRT & & \\
Técnica 2D & 1155 & \\
Técnica de radioterapia & & \\
estereotáxica fraccionada & 9 & \\
Total de pacientes por técnica & & 316 \\
Total de correcciones & & 73 \\
Proyección antero-posterior & 347 & \\
Proyección lateral izquierda & 351 & \\
Proyección tangencial interna & 90 & \\
Proyección antero-posterior & & \\
de fosa & 7 & \\
Proyección postero-anterior & 4 & \\
Proyección oblicua & 10 & \\
Total de imágenes tomadas & & 809 \\
\hline
\end{tabular}

3D CRT: radioterapia conformal tridimensional; IMRT: radioterapia de intensidad modulada; 2D: bidimensional.

El Departamento de Radioterapia del Centro Javeriano de Oncología tiene una dotación de inmovilizadores y accesorios para la reproducibilidad diaria del posicionamiento de los pacientes, los cuales se enumeran a continuación:

- Icopor de alta densidad con medidas de 20 × $20 \mathrm{~cm}$.

- Plano inclinado en fibra de carbono o acrílico con soportes de brazo y cabeza.

- Soporte de cabeza y cuello ref. AB- C- D- E- F.

- Base acrílica para cabeza y cuello.
- Máscaras termoplásticas para técnica 3D CRT (radioterapia conformal tridimensional) e IMRT (radioterapia de intensidad modulada).

- Fijadores de cabeza y hombros en fibra de carbono.

- Inmovilizador de extremidades.

Para la verificación de la forma y localización del posicionamiento, el departamento cuenta con:

- Sistema portal iview (paneles de silicio amorfo) que viene incorporado en uno de los aceleradores lineales (Elekta) empleados en la administración de tratamientos. Este sistema nos permite adquirir las imágenes en tiempo real.

- Sistema de adquisición de imágenes digitales Kodak.

- Película radiográfica para megavoltaje.

En nuestro Departamento de Radioterapia, tenemos dos aceleradores lineales para la administración de tratamientos, los cuales están referenciados como SLI y PRECISE de marca ELEKTA los cuales tienen energías en las modalidades de fotones $(4,8$ y 18 $\mathrm{MV}$, y 6 y $15 \mathrm{MV}$, respectivamente) y electrones $(6,8,10,12 \mathrm{y}, 15 \mathrm{MeV}, \mathrm{y} 6$, 9, 12,15 y $18 \mathrm{MeV}$, respectivamente).

Para el proceso de simulación, contamos con un tomógrafo TOSHIBA, 
SCANNER ASTEION modelo TSX021B. Este es un tomógrafo helicoidal que consta de cuatro detectores, una camilla plana adaptada para radioterapia la cual soporta un peso máximo de $330 \mathrm{~kg}$, y un sistema de láseres incorporados al sistema tomográfico que permiten mover o marcar al paciente en tres coordenadas espaciales; éste es de $28 \mathrm{~cm}$ de diámetro. Este equipo se encuentra interconectado mediante red DICOM al TPS Oncentra Masterplan.

Se diseñó un instrumento de medición para estimar los factores empleados en nuestro estudio (correcciones por posicionamiento, técnica de tratamiento) que consiste en un formato de registro descrito en el anexo 1.
Durante la simulación, se les dejan a los pacientes unos puntos de referencia que sirven para la ubicación exacta del paciente en el equipo de tratamiento.

A continuación se describe la técnica que empleamos en nuestro centro para establecer estos puntos de referencia, denominados tatuajes de tratamiento.

\section{Técnica para realizar los tatuajes}

Los tatuajes son las marcas de referencia que se usan para ubicar al paciente. Son de tinta de color para evitar que se caigan con el baño o el roce con la ropa del paciente, y se ven como pequeños lunares sobre la piel.

1. En el sitio de ubicación del tratamiento o referencia de simulación, previamente marcado, se coloca una gota de tinta en la piel.

2. Se introduce el bisel de una aguja de calibre y punta finos, y se comienza a romper la piel sobre la gota de tinta.

3. Se limpia el área anatómica.
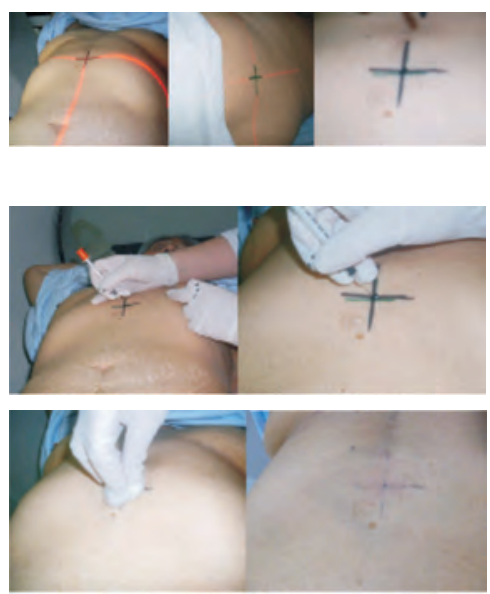

4. Se revisan el tamaño y la reproducibilidad del tatuaje.

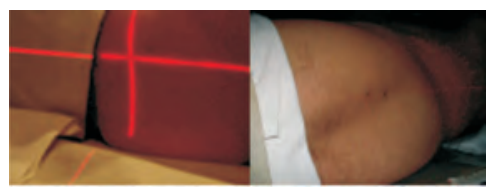

Figura 1. Procedimiento para hacer tatuajes. 


\section{Control de calidad y seguimiento de la administración de tratamientos}

Al iniciar la radioterapia, el paciente se posiciona sobre la mesa de tratamiento del acelerador lineal. Una vez localizado el punto de referencia o tatuajes de simulación, se mueven coordenadas según la planeación del tratamiento, para localizar exactamente el área anatómica que se va a tratar.

Una vez realizado este procedimiento, se toman las imágenes radiológicas necesarias para corroborar la ubicación del isocentro, con respecto a las radiografías reconstruidas digitalmente (Digitally Reconstructed Radiograph, DRR) durante la planeación. Éstas se toman al principio y durante el transcurso del tratamiento, como método de control de calidad que permite seguridad en la reproducibilidad diaria del posicionamiento del paciente y la cobertura del volumen blanco.

Este control de calidad, se hace con el sistema de coordenadas X, Y y Z (figura 2), para comparar las imágenes tomadas en el equipo de tratamiento y las enviadas. Sistema definido de la siguiente manera:

\section{- EJE Z}

Representa la coordenada vertical, formando un plano que divide al cuerpo en anterior y posterior.

\section{- EJE Y}

Representa la coordenada longitudinal, formando un plano que divide al cuerpo en superior e inferior.

\section{- EJE X}

Representa la coordenada lateral, formando un plano que divide al cuerpo en derecho e izquierdo

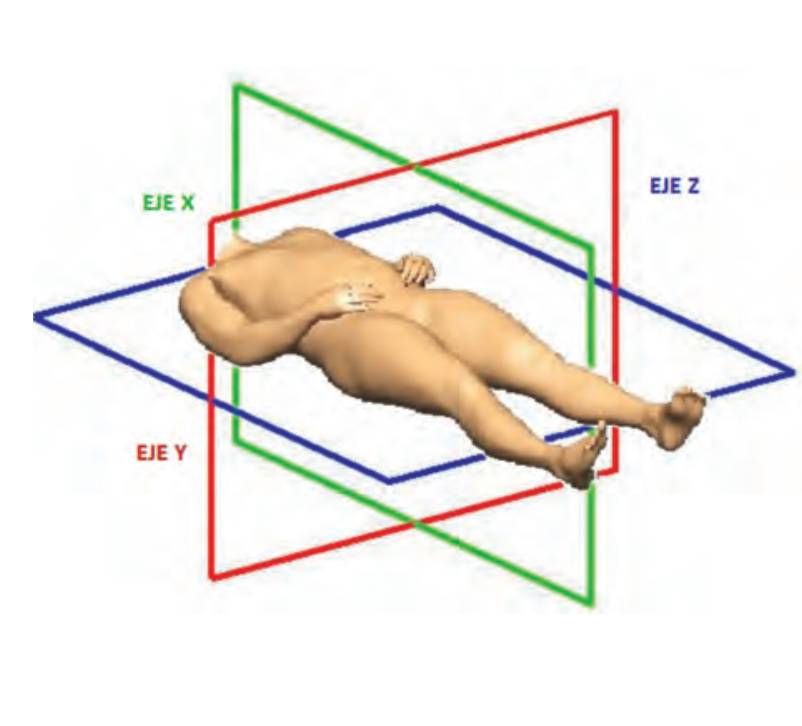

Figura 2. 
La técnica que se emplea para la toma de las imágenes es de suma importancia, ya que de ella también depende en gran manera la calidad de la imagen para diferenciar los límites del campo que serán comparados con respecto a estructuras óseas que se evalúan para verificación del posicionamiento del paciente. En el caso de los aceleradores, los parámetros técnicos empleados en la toma de radiografías, son la unidad monitor (UM), o sea, la unidad funcional del equipo de tratamiento. Las técnicas empleadas son:

Tabla 2. Técnica empleada para la toma de imágenes con megavoltaje

\begin{tabular}{lrr}
\hline & Campo abierto & Campo de referencia \\
\hline Proyección antero-posterior & & \\
Próstata, cráneo, tórax & $2 \mathrm{UM}$ & $4 \mathrm{UM}$ \\
Cuello uterino, recto & $2 \mathrm{UM}$ & $2 \mathrm{UM}$ \\
Abdomen & $3 \mathrm{UM}$ & $5 \mathrm{UM}$ \\
Extremidades & $2 \mathrm{UM}$ & $2 \mathrm{UM}$ \\
& & \\
Proyección lateral & & \\
Próstata & $6-8 \mathrm{UM}$ & $7-9 \mathrm{UM}$ \\
Cráneo & $2 \mathrm{UM}$ & $4 \mathrm{UM}$ \\
Cuello uterino, recto & $5-7 \mathrm{UM}$ & $6-7 \mathrm{UM}$ \\
Abdomen, tórax & $4-6 \mathrm{UM}$ & $6-7 \mathrm{UM}$ \\
& & \\
Proyección tangencial & & \\
Seno & $2 \mathrm{UM}$ & $2-3 \mathrm{UM}$ \\
Tórax & $3 \mathrm{UM}$ & $4-5 \mathrm{UM}$ \\
\hline
\end{tabular}

UM: unidad monitor.

\section{Método de evaluación de los criterios para determinar las correcciones por posicionamiento}

Para hacer el control de calidad de cada una de las áreas anatómicas para incertidumbres por posicionamiento, tuvimos en cuenta los siguientes crite- rios de evaluación, los cuales se correlacionaron con la imagen de reconstrucción digital.

En el caso de la próstata se tuvieron en cuenta las referencias anatómicas para la evaluación de las imágenes iniciales y de control (figuras 3-8). 


\section{Proyección antero-posterior}

IMAGEN DRR

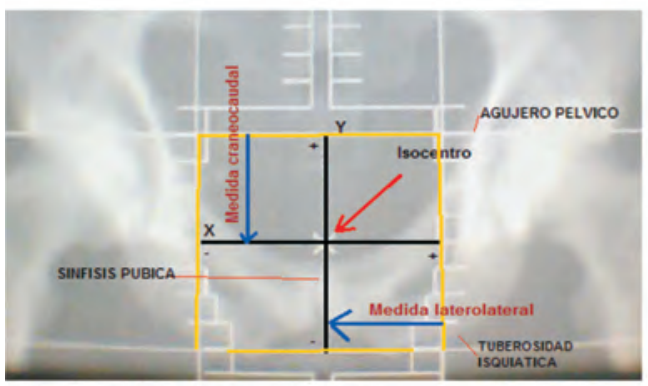

IMAGEN DE CONTROL

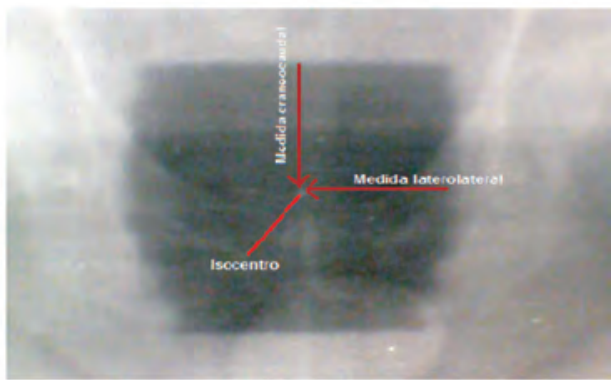

Figura 3. A la izquierda, imagen digital, y a la derecha, imagen tomada a $0^{\circ}$.

\section{Proyección lateral izquierda}

IMAGEN DRR

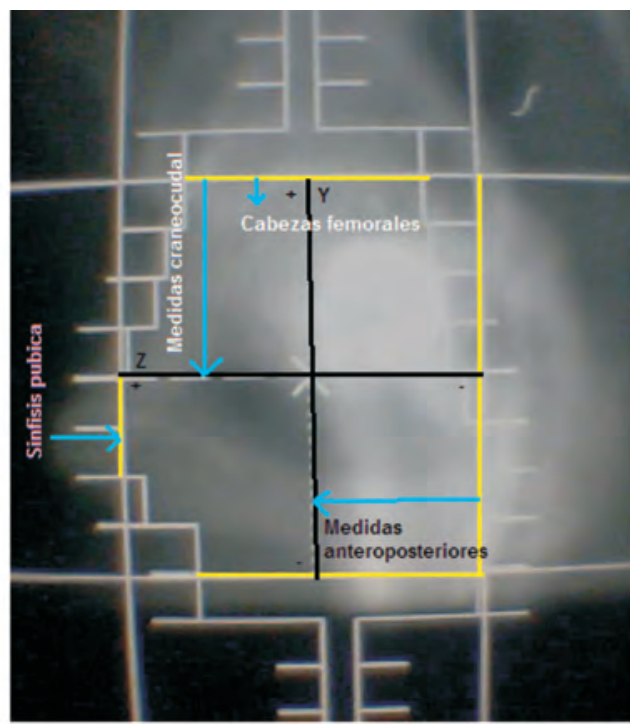

IMAGEN DE CONTROL

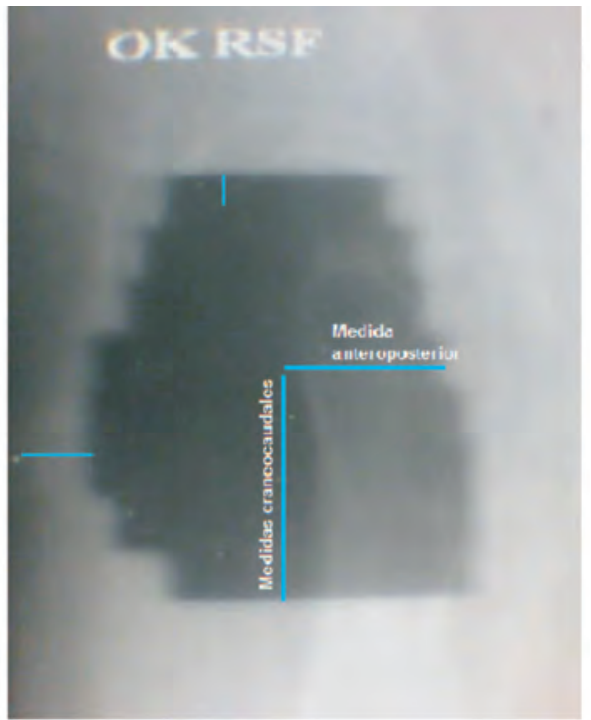

Figura 4. A la izquierda, imagen digital, y a la derecha, imagen tomada con un ángulo de $90^{\circ}$. 


\section{Proyección tangencial}

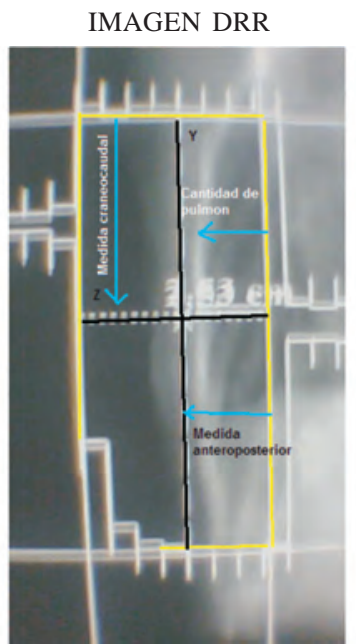

IMAGEN DE CONTROL

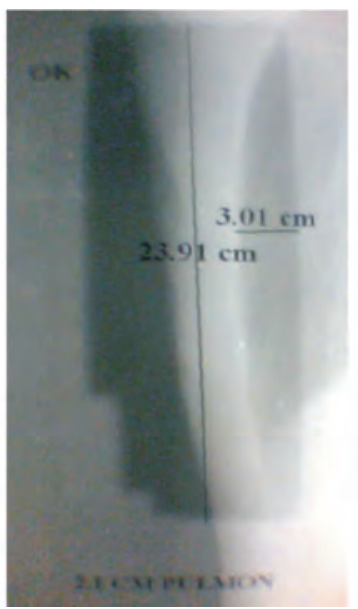

En pacientes con mastectomía sin reconstrucción, se tuvo en cuenta el desborde del campo con respecto a la piel; para evaluar el posicionamiento, comparamos el ángulo del húmero en relación con la DRR (imagen de reconstrucción digital).

Figura 5. A la izquierda, imagen digital, y a la derecha, imagen en proyección tangencial.
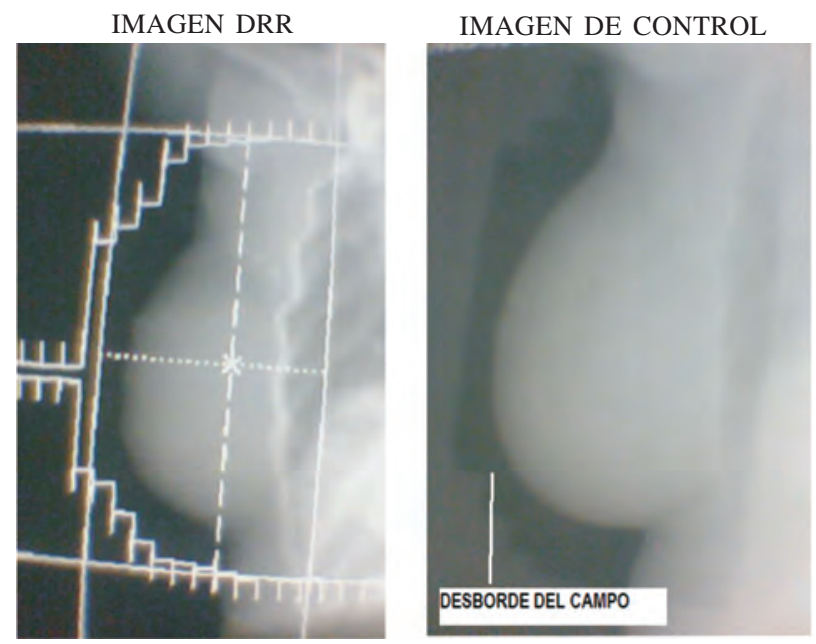

Figura 6. A la izquierda, imagen digital, y a la derecha, proyección tangencial con demostración del desborde de campo.

Para evaluar la cantidad de pulmón, se emplea la fórmula de desmagnificación, medición del campo en $\mathrm{X}$ ó $\mathrm{Y}$ multiplicado por la medida que arroje el sistema de imágenes, dividido por la cantidad de pulmón observada en la imagen de control, y se compara con la cantidad de pulmón establecida en la DRR (figuras 5 y 6). 
Cabeza y cuello

Proyección antero-posterior

IMAGEN DRR

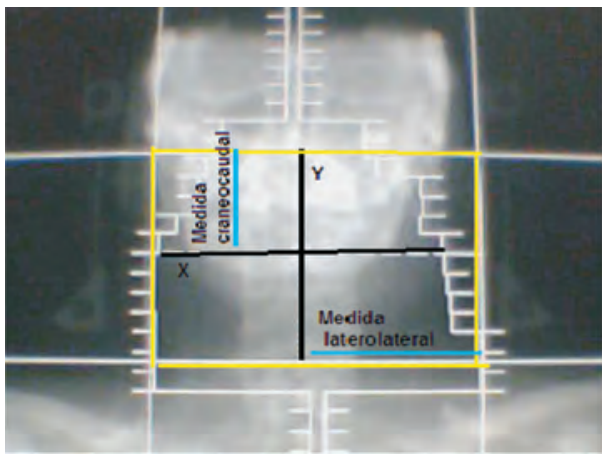

IMAGEN DE CONTROL

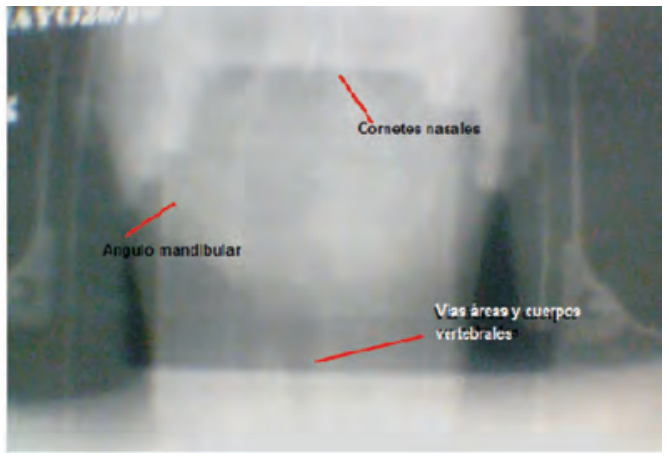

Figura 7. A la izquierda, imagen digital, y a la derecha, imagen tomada a $0^{\circ}$.

\section{Proyección lateral izquierda}

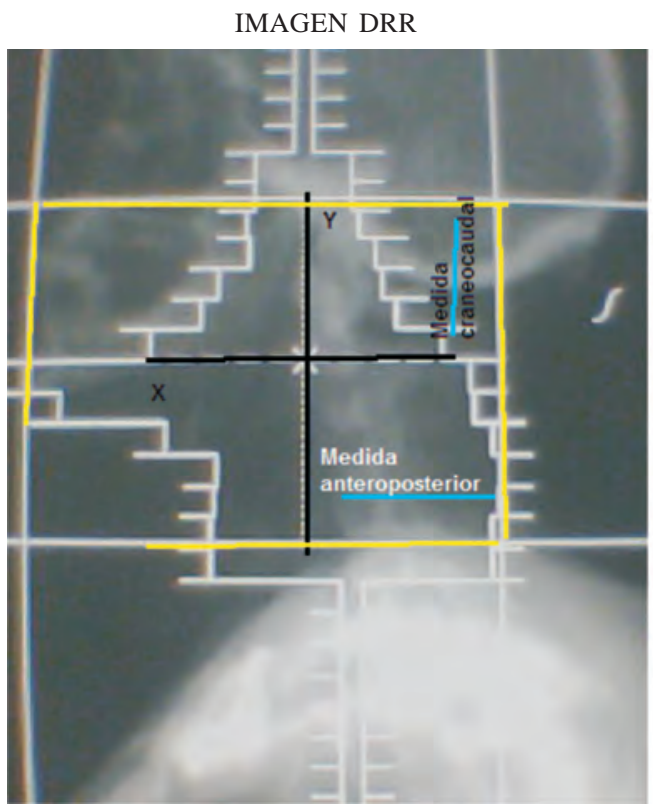

IMAGEN DE CONTROL

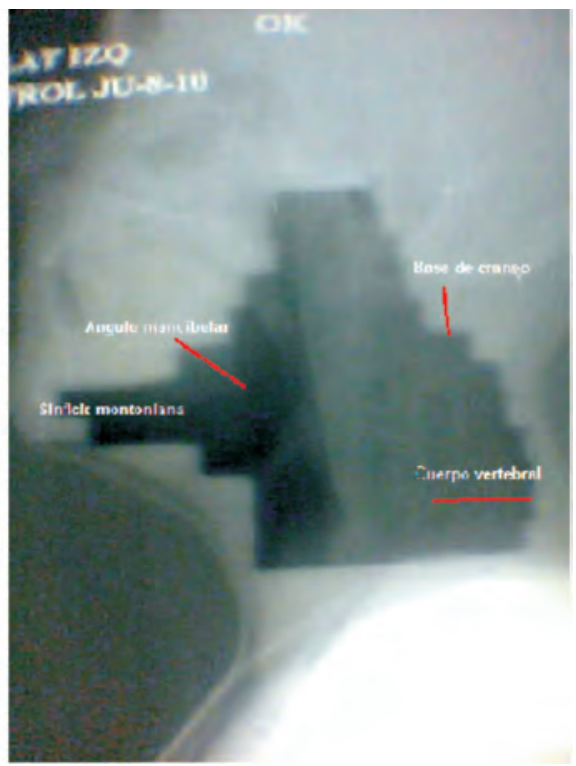

Figura 8. A la izquierda, imagen digital, y a la derecha, imagen tomada con ángulo de $90^{\circ}$. 


\section{Resultados}

Se analizó un total de 316 pacientes en el periodo comprendido entre el 17 de marzo y el 18 de junio de 2010, de los cuales los tumores más frecuentes se muestran en la tabla 3 .

\section{Tabla 3. Localización} de los tumores

\begin{tabular}{lr}
\hline Tumores & Total \\
\hline Próstata & 83 \\
Mama & 68 \\
Sistema nervioso central (primario) & 37 \\
Metástasis & 32 \\
Cabeza y cuello & 23 \\
Ginecológico & 19 \\
Abdomen & 16 \\
Pelvis (primarios) & 12 \\
Tórax & 11 \\
Recto & 9 \\
Extremidades (sarcomas) & 6 \\
Total & 155 \\
\end{tabular}

Tabla 4. Finalidad del tratamiento
Finalidad

Frecuencia

Curativa

Paliativa

26

Total
En el tiempo del estudio, encontramos que la mayoría de los tratamientos tuvieron intención curativa (figura 9) (tabla 5).

\section{Tabla 5. Técnica de tratamiento}

Técnica de tratamiento

Total

3D CRT

241

IMRT

55

Convencional 2D

11

Radioterapia estereotáxica fraccionada 9

Total

3D CRT: radioterapia conformada tridimensional; IMRT: radioterapia de intensidad modulada; D: bidimensional.

\section{Tumores}
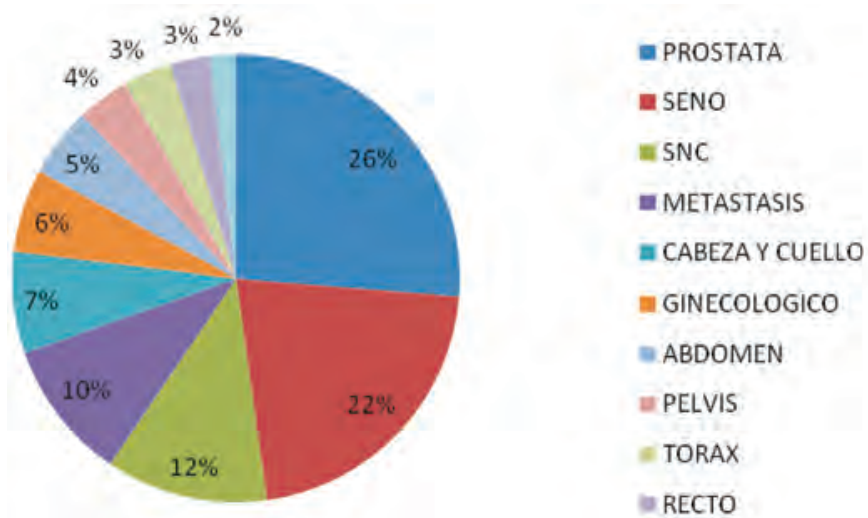

Figura 9. Porcentajes por localización de tumores. 
Según la técnica, encontramos que $76 \%$ de los pacientes fueron tratados con 3D CRT y $17 \%$, con IMRT, siendo estas las técnicas más modernas para el tratamiento de las enfermedades oncológicas (figura 10) (tabla 6).

Tabla 6. Adquisición de imagen

Película radiográfica

CR-Kodak

169

640

Total de imágenes de los pacientes en los equipos de tratamiento, se emplearon las técnicas de imágenes radiológicas que nos permiten verificar este ítem. Del total de imágenes obtenidas en el estudio, $21 \%$ se registró en película radiográfica y, $79 \%$, en imagen digital (CR-Kodak) (figura 11) (tabla 7).

Tabla 7. Número de correcciones por eje

X $\quad$ Y $\quad$ Z

Total 25 22 26

Finalidad

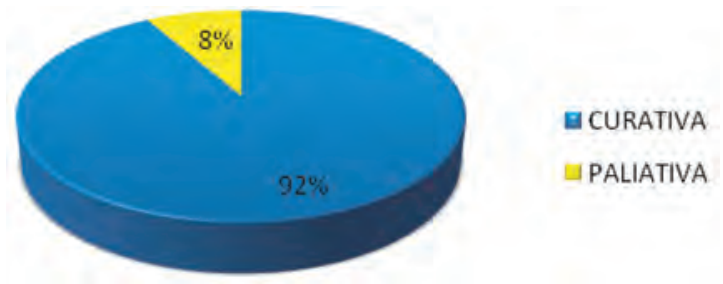

Figura 10. Porcentajes según finalidad del tratamiento.

\section{4\% Técnica de tratamiento}

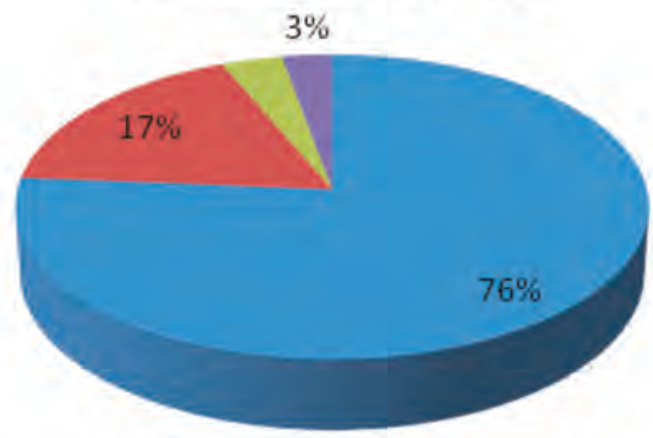

T.30CRT $=$ T.IMRT $=T .20=$ RTESTEREOTAXICA FRACCIONADA

Figura 11. Porcentajes y cantidades según técnica de tratamiento. 
Adquisición de imagen

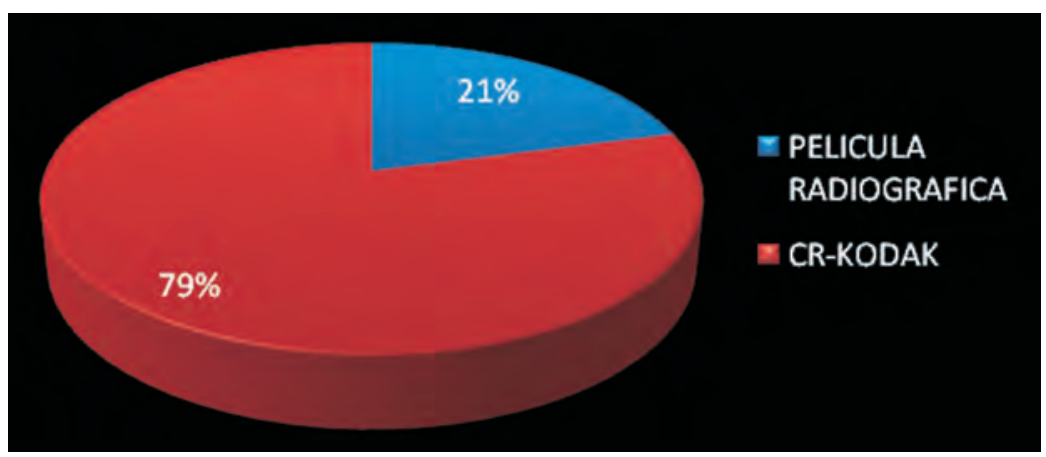

Figura 12. Representación en porcentajes del tipo de adquisición de imagen.

Se hicieron 809 proyecciones (figura 13).

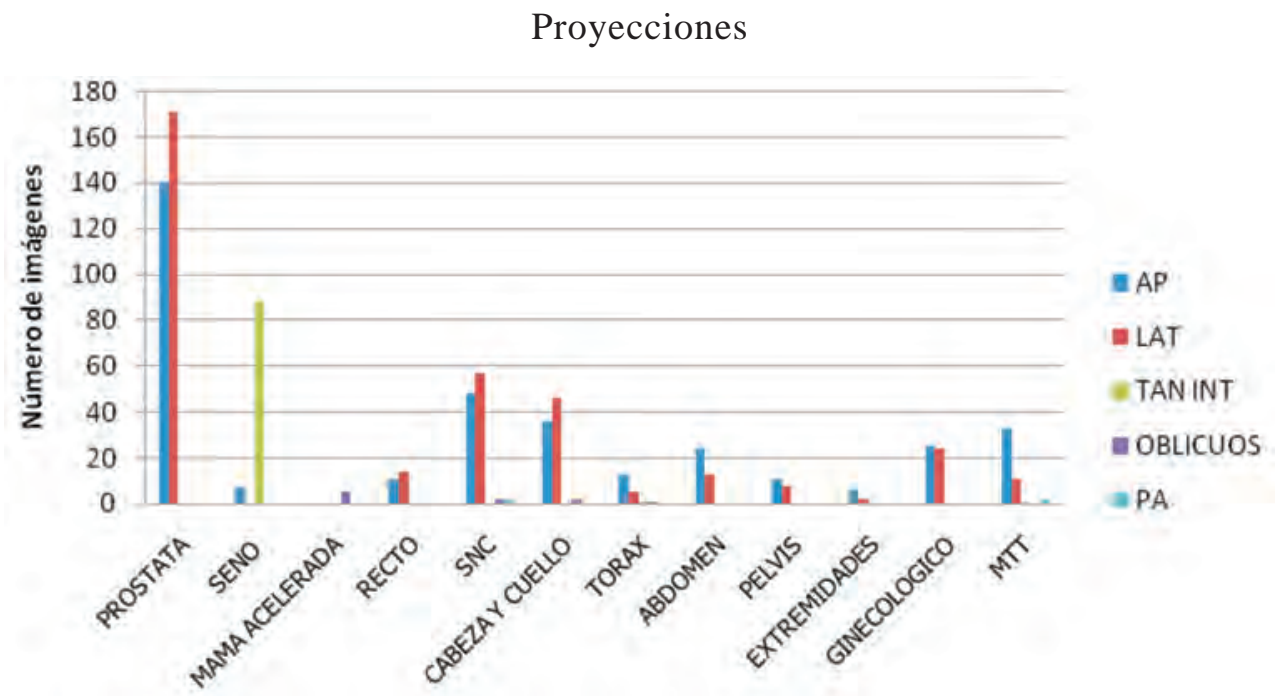

Figura 13. Tipo de proyecciones tomadas.

\section{Análisis estadístico de las correcciones}

Del total de las imágenes realizadas, 736 no requirieron corrección y 73 imágenes presentaron correcciones por posicionamiento (figura 14). 
Imágenes tomadas

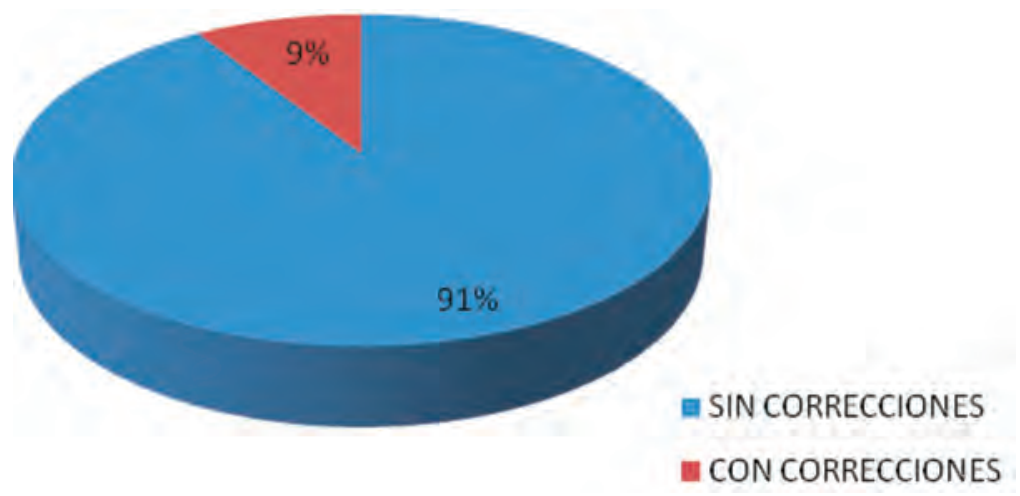

Figura 14

Para el análisis estadístico, se tuvieron en cuenta el promedio y la desviación estándar, que es una medida empleada para determinar la dispersión de los datos tabulados con respecto al valor promedio de la distribución, lo que permite calcular cuánto tienden a alejarse los valores del promedio.

A continuación se describen los resultados obtenidos para cada eje, con respecto a las correcciones hechas en el estudio (figuras 15-17).

\section{Correcciones en el eje $\mathrm{Y}$}

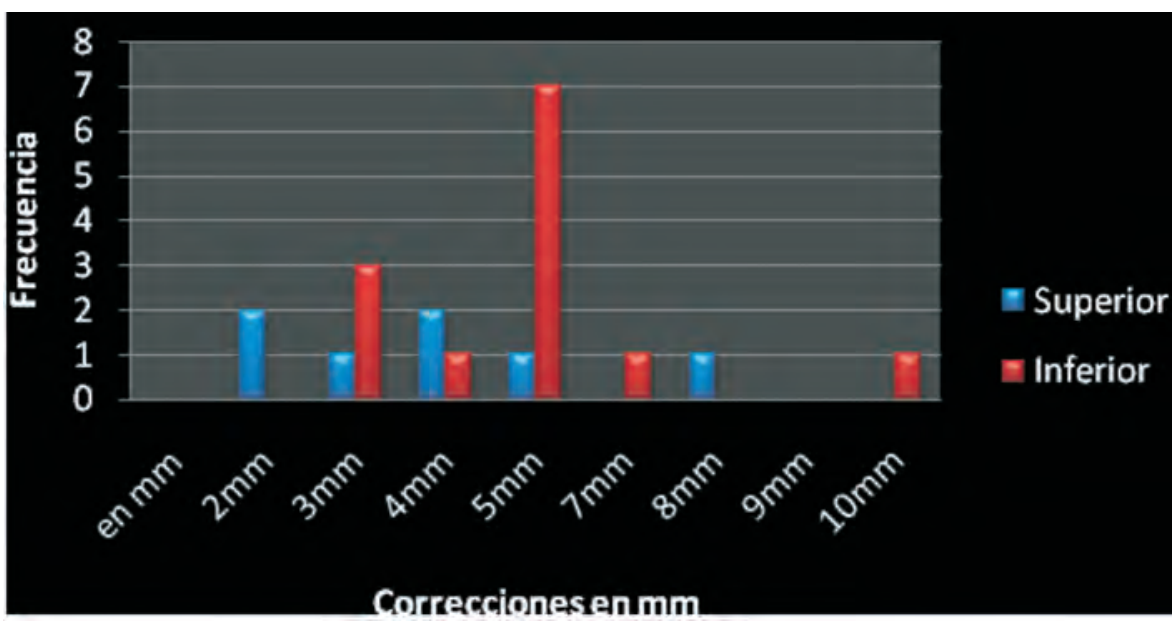

Figura 15. Promedio de 4,5 mm, con desviación estándar de 1,95 mm. 
Correcciones en el eje Z

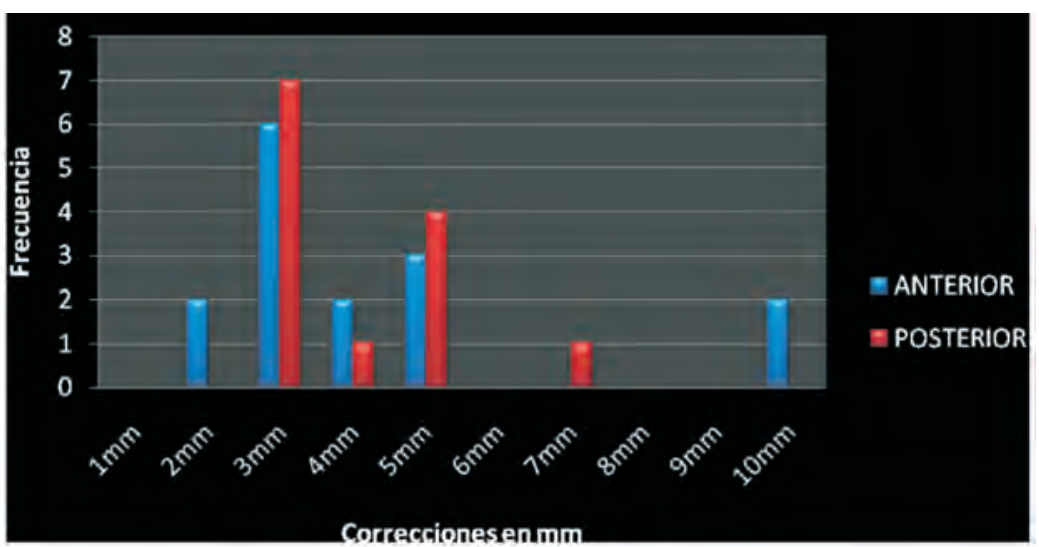

Figura 16. Promedio de $4 \mathrm{~mm}$, con desviación estándar de $2 \mathrm{~mm}$.

\section{Correcciones en el eje $\mathrm{X}$}

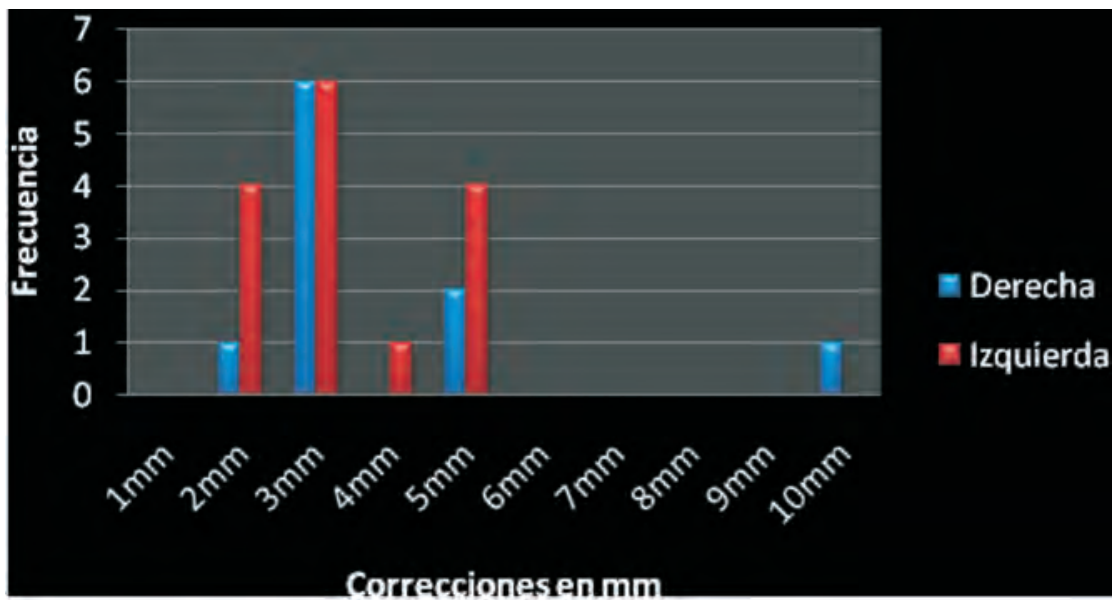

Figura 17. Promedio de $3,6 \mathrm{~mm}$, con desviación estándar de 1,7 mm. 
Los porcentajes correspondientes a cada eje se muestran en la figura 18 .

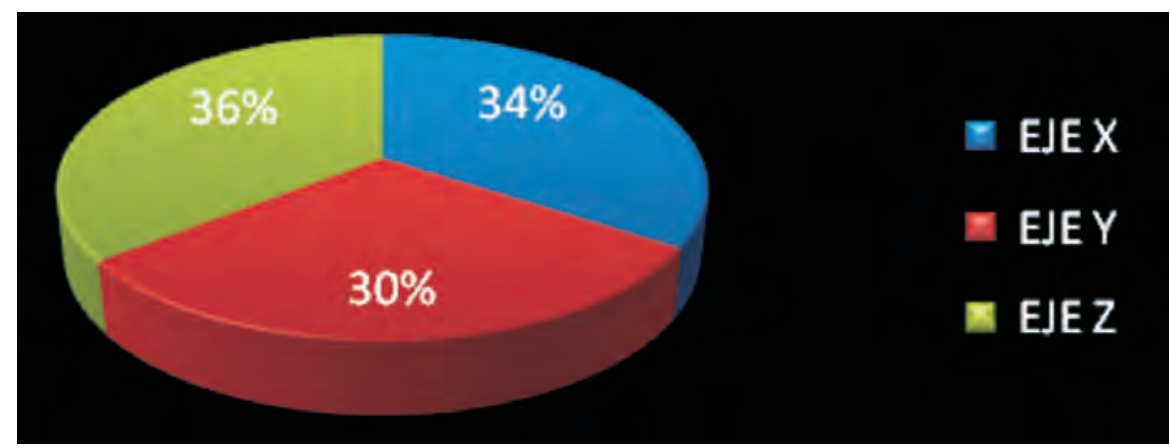

Figura 18

De las correcciones hechas en los pacientes, el mayor porcentaje correspondió al cáncer de próstata (59\%).

Para cáncer de próstata, se tomó un total 140 imágenes en la proyección antero-posterior y de 171 en la proyección lateral; para esta neoplasia maligna se presentaron 43 correcciones.

El análisis de las correcciones de 3 $\mathrm{mm}$ o menos y de más de $3 \mathrm{~mm}$, en pacientes con cáncer de próstata, se muestra a continuación (figura 19) (tabla 9).

Tabla 8. Correcciones en próstata

\begin{tabular}{llcc}
\hline & Sentido & $\leq \mathbf{3} \mathbf{~ m m}$ & $>\mathbf{3} \mathbf{~ m m}$ \\
\hline Eje X & Derecha & 4 & 0 \\
& Izquierda & 8 & 1 \\
Eje Y & Superior & 2 & 2 \\
& Inferior & 3 & 3 \\
Eje Z & Anterior & 6 & 4 \\
& Posterior & 6 & 4 \\
& Totales & 29 & 14 \\
\hline
\end{tabular}

Correcciones en próstata

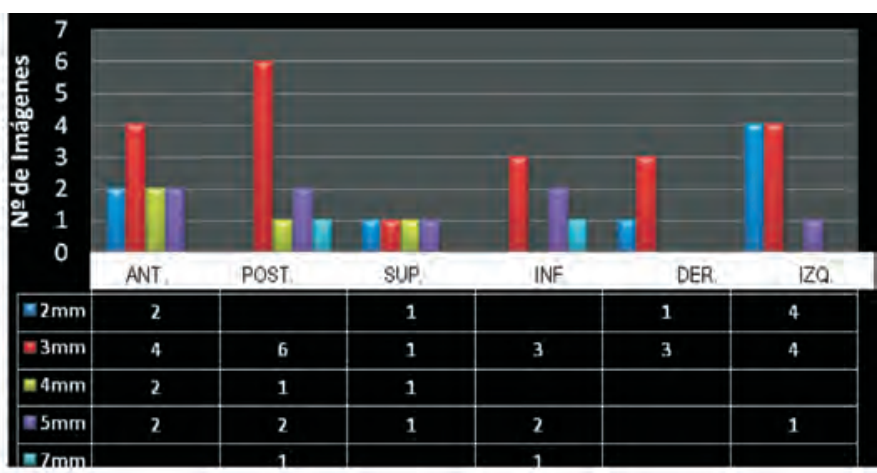

Figura 19 


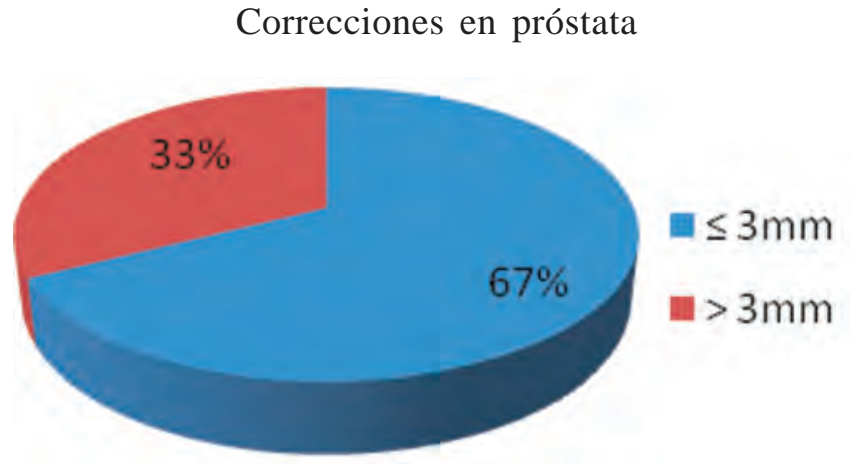

Figura 19a. Porcentajes de correcciones de $3 \mathrm{~mm}$ o menos y de más de $3 \mathrm{~mm}$.

Histograma de correcciones en próstata

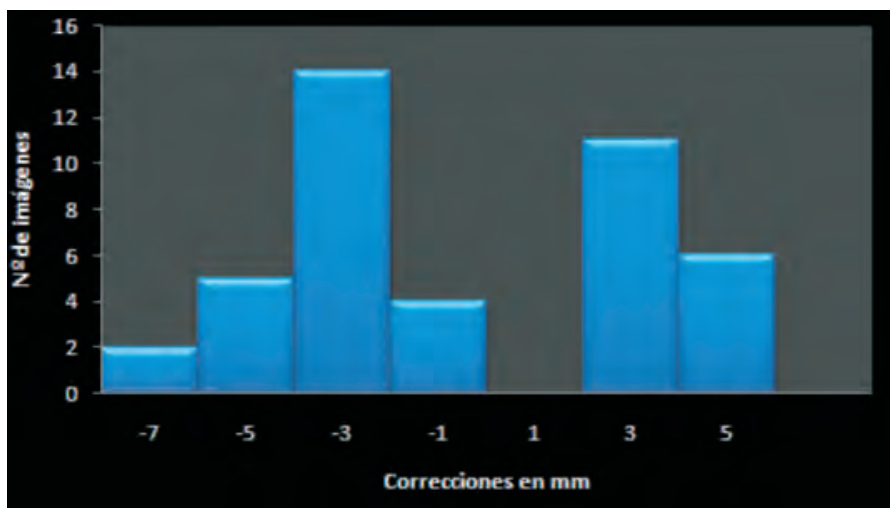

Figura 19b. Histograma.

Tabla 9. Representación gráfica de las correcciones en próstata, en los tres ejes espaciales

\begin{tabular}{llcc}
\hline & & \multicolumn{2}{c}{ Correcciones en próstata } \\
\hline Eje $\mathrm{X}$ & Sentido & $\leq \mathbf{3} \mathbf{m m}$ & $\mathbf{3} \mathbf{m m}$ \\
\cline { 2 - 3 } Eje Y & Derecha & 4 & 0 \\
& Izquierda & 8 & 1 \\
\multirow{2}{*}{ Eje Z } & Superior & 2 & 2 \\
& Inferior & 3 & 3 \\
& Anterior & 6 & 4 \\
\hline & Posterior & 6 & 4 \\
\hline
\end{tabular}




\section{Discusión}

En un número importante de estudios se ha descrito la reproducibilidad del posicionamiento del paciente durante el tratamiento de radioterapia [1-6]. En éste se incluyen todas las enfermedades oncológicas tratadas en el Centro Javeriano de Oncología. La más frecuente fue el cáncer de próstata, en la que se presentó la mayor cantidad de correcciones por posicionamiento.

En estos pacientes, el promedio de las correcciones en los tres ejes espaciales fue de $3,4 \mathrm{~mm}$, similar a lo reportado en otros estudios[7-9]. Sin embargo, observamos variaciones de $5 \mathrm{~mm}$ o más en pacientes con sobrepeso. Wons et al. (10) encontraron una significante incertidumbre en el posicionamiento en la dirección lateral (eje X) en 329 pacientes con cáncer de próstata.

En este estudio se encontró que los pacientes con pérdida de peso importante debida a la enfermedad oncológica y la toxicidad del tratamiento, presentaron mayor frecuencia de errores por posicionamiento debido a la rotación de la pelvis; sin embargo, no fue específicamente analizado, pero podría ser considerado en un futuro.

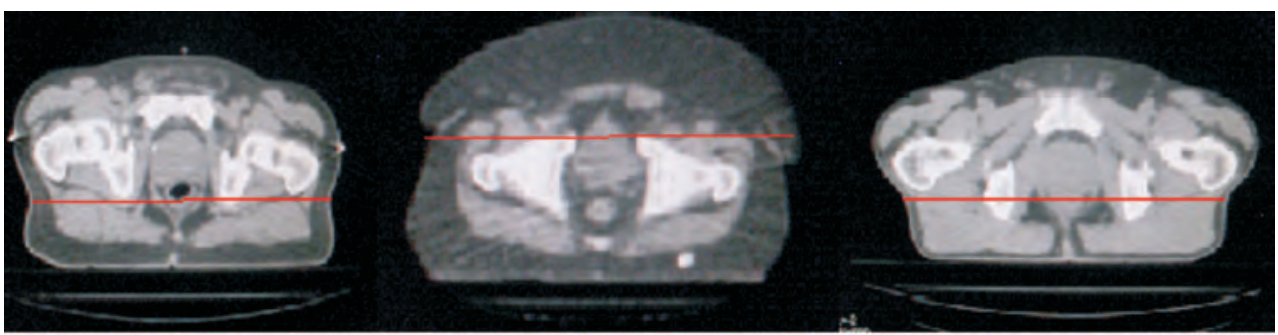

Pacientes con poca Pacientes con abundante tonicidad muscular, estos pacientes hacen que la pelvis al localizarse quede basculada. tejido adiposo, que impide ubicar correctamente la marca de referencia lateral, debido a que el abdomen es muy inestable.

Figura 20. Representación gráfica de las características de los pacientes para tratamiento de cáncer de próstata. 
En el protocolo empleado en el centro para pacientes con cáncer de próstata, el margen que damos al volumen blanco clínico (clinical target volume, $\mathrm{CTV}$ ) es de $1 \mathrm{~cm}$ en sentido anterior, superior, inferior, izquierdo y derecho, y de $7 \mathrm{~mm}$ en sentido posterior (PTV); estos márgenes son adecuados para asegurar el cubrimiento de la dosis, teniendo en cuenta no sólo las incertidumbres por posicionamiento, sino los movimientos de los órganos interfracción e intrafracción.

En otras neoplasias diferentes al cáncer de próstata, los errores de posicionamiento fueron menores en frecuencia y los encontrados se debieron al regular estado general del paciente debido a pérdida de peso, dolor, alteración neurológica, etc.

En algunas pacientes con cáncer de mama, las modificaciones se hicieron con relación a la abducción del brazo, en muchos casos debido a la falta de terapia física después del vaciamiento axilar.

\section{Conclusión}

Los datos encontrados en el estudio muestran una adecuada reproducibilidad del posicionamiento en las diferentes enfermedades oncológicas tratadas en nuestro servicio de radioterapia. Sin embargo, se observó que en pacientes con cáncer de próstata, en especial obesos y delgados, podría ser necesaria la implementación de inmovilizadores de extremidades inferiores, con la finalidad de asegurar la relajación de la musculatura pélvica y así evitar que esta región anatómica quede basculada. Otra medida recomendada es colocar marcas radiopacas (fiducias) tanto en la base como en el vértice de la glándula prostática, lo cual permitirá realizar un mejor control de calidad en cuanto a incertidumbres por posicionamiento, tanto del paciente como de la próstata. Podrán realizarse futuros estudios teniendo en cuenta la estimación de incertidumbres por posicionamiento mediante el uso de los inmovilizadores recomendados y la colocación de fiducias.

\section{Abreviaciones}

- 3D CRT:3 dimensional conformal radiation therapy

- IMRT: intensity modulated radiation therapy

- CTV: clinical target volume

- PTV: planning target volume

- DRR: digital reconstructed radiograph

\section{Bibliografía}

1. Ploeger LS, Frenay M, Betgen A, van Herk M. Application of video imaging for improvement of patient set-up. Radiother Oncol. 2003;68:277-84.

2. Bert C, Metheany KG, Doppke KP, Powell SN. Clinical experience with a 3D surface patient setup system for alignment of partial-breast irradiation patients. Int J Radiat Oncol Biol Phys. 2006;64:1265-74. 
3. Krengli M, Gaiano S, Mones E, Beldi D, Loi G. Reproducibility of patient setup by surface image registration system in conformal radiotherapy of prostate cancer. Radiation Oncol. 2009;4:1-10.

4. Serago EF, Buskirk SJ, Gale A, Earle J. Comparison of daily megavoltaje electronic portal imaging or kilovoltaje imaging with marker sedes to ultrasound imaging or skin mars for prostate localization and treatment positioning in patients with prostate cancer. Int J Radiat Oncol Biol Phys. 2006;65:1585-92.

5. Cranmer G. A treatment planning investigator into the dosimetric effects of systematic prostate patient rotational set-up errors. Med Dosim. 2008; 33:199-205.

6. Gordon J, Crimaldi A, Hagan M, Siebers J. Evaluation of clinical margins in simulation of patient setup errors in prostate IMRT treatment plans. Med Phys. 2007;34:202-14.
7. Kupelian PA, Lee C, Meeks SL. Evaluation of image-guidance strategies in the treatment of localized prostate cancer. Int J Radiat Oncol Biol Phys. 2008;70:1151-7.

8. Hurkmans CW, Remeijer P, Leberque J, Mirjnheer B. Set-up verification using portal imaging; review of current clinical practice. Radiother Oncol. 2001;58:105-20.

9. Fiorino C, Reni M, Bolognesi A, Calandrino R. Set-up error in supinepositioned patients immobilized with two different modalities during conformal radiotherapy of prostate cancer. Radiother Oncol. 1998; 49:133-41.

10. Wong JR, Gao Z, Uematsu M, Merrick $\mathrm{S}$, Cheng $\mathrm{CW}$. Interfractional prostate shifts: Review of 1870 computed tomography (CT) scans obtained during image-guided radiotherapy using CTon rails for the treatment of prostate cancer. Int J Radiat Oncol Biol Phys. 2008;72:1376-401. 